\title{
Real - Time Tomographic Reconstructor based on Convolutional Neural Networks for Solar Observation
}

\author{
Fernando Sánchez Lasheras ${ }^{1}$, Celestino Ordóñez ${ }^{2}$, Javier Roca-Pardiñas ${ }^{3}$, Francisco \\ Javier de Cos Juez ${ }^{2}$ \\ ${ }^{1}$ Department of Mathematics, Faculty of Sciences, University of Oviedo, 33007 Oviedo, Spain \\ ${ }^{2}$ Exploitation and Prospecting Department, University of Oviedo, 33004 Oviedo, Spain \\ ${ }^{3}$ Department of Statistics and Operations Research, University of Vigo, 32608 Vigo, Spain
}

Corresponding author: E-mail address: sanchezfernando@uniovi.es (F. Sánchez Lasheras)

\begin{abstract}
Solar observation is the branch of astronomy devoted to the study of the Sun. Tomographic reconstruction is a kind of multidimensional inverse problem where the challenge is to yield an estimate of a specific system from a finite number of projections. When the light wave-front that comes from the Sun penetrates the atmosphere, it suffers some distortions caused by optically turbulent layers present at different altitudes. This turbulence changes the wave-front's shape and morphology. Therefore, in order to obtain a good-quality image, it is necessary to correct the induced error. This is done by applying adaptive optics (AO) techniques. In the case of the present research, it is performed with the help of a Multi-Conjugate Adaptive Optics System (MCAO). The reconstruction technique proposed in this research is a MCAO based on Convolutional Neural Networks (CNN). This research develops and assesses a real-time tomographic reconstructor based on convolutional neural networks, able to correct the error introduced by the atmosphere in the light wave-front received from the Sun. The CNN was trained and validated using data from the Durham AO Simulation Platform as input information. This Platform has been specifically adapted for this project in order to simulate a solar telescope. A learning algorithm able to train the CNN was developed and the CNN reconstructor results validated in the simulated environment. As far as the authors know, this is the first time that an AO system based on CNN has been developed for solar telescopes.
\end{abstract}

Keywords: solar activity, turbulence, tomography, deep learning, convolutional neural networks

\section{Introduction.}

The Sun is the nearest star to Earth, being some 148 million kilometres distant at its closest approach. The sunlight that reaches Earth is eight minutes old, as this is the time required to travel from the Sun to our planet. Despite the distance, and due to its size, seen from Earth, the Sun is one of the largest objects in the sky. 
The Sun has a bright surface called the photosphere. Sometimes dark patches called sunspots are observed on its surface. Some of these sunspots are so large that they can be observed with the naked eye, but most of them cannot. Sunspots are magnetic regions formed where a number of concentrated field lines emerge through the photosphere to form a region of inward-directed or positive fields.

Since the mid-19th century the link between the solar cycle, sunspots and some terrestrial phenomena such as aurorae has been recognised. Nowadays, solar observation is carried out with the help of solar telescopes. These operate during the day, which makes their use more complicated than that of telescopes devoted to night observation, due to the turbulence caused by the sun heating the atmosphere. Another problem specific to solar telescopes is the heating they suffer owing to their direct exposure to the rays of the sun.

One of the earliest systematic solar observations with scientific purposes was the one made by The Royal Observatory of the Spanish Navy located at Cadiz (Spain), where a solar telescope was installed in 1832 [1]. The results of these studies were made available from 1835. The first photograph of the Sun was taken in France in 1842 by Lerebours, while the first systematic series of solar photographs was taken from 1858 to 1872 by the British astronomer De la Rue [2].

Since those early days, interest in solar observation has grown enormously. Nowadays solar observation is the branch of astronomy devoted to the study of the Sun and its behaviour. One of its aims is to discover how solar activity is created and how space and Earth weather results from that activity. Solar observation helps us to understand the physics behind the activity displayed by the Sun's atmosphere, which drives space weather in the heliosphere and on the planets of our solar system.

At the time when these lines are being written, the new European Solar Telescope (EST) is under development. This device will be optimized for studies of magnetic coupling between the deep photosphere and upper chromosphere. The EST will employ different instruments at the same time in order to generate 2D spectral information.

\section{Materials and Methods.}

\subsection{Tomographic reconstruction.}

Tomographic reconstruction [3] is a kind of multidimensional inverse problem where the challenge is to yield an estimate of a specific system from a finite number of projections. Fig. 1 shows a sketch of a parallel beam geometry employed in tomography and tomographic reconstruction. In this Figure, each projection, resulting from tomography under a specific angle, is made up of the set of lines integral through the object. 
The intensity received can be expressed as follows:

$$
I=I_{0} \cdot e^{-\int \mu(x, y) \cdot d x}
$$

Data to be collected as a series of parallel rays, at position, across projection angle $\theta$. Where $\mu$ is the attenuation coefficient as a function of position (Radon Transform) and $I_{0}$ the original intensity. The total attenuation $p$ of a ray at position $\theta$ has the following equation:

$$
p_{\theta}(r)=\ln \left(\frac{I}{I_{0}}\right)=-\int \mu(x, y) \cdot d s
$$

Please note that:

$$
r=x \cdot \cos \theta+y \cdot \sin \theta
$$

The equation expressed above can be rewritten as follows:

$$
p_{\theta}(r)=\int_{-\infty}^{\infty} \int_{-\infty}^{\infty} \mu(x, y) \cdot \delta(x \cdot \cos \theta+y \cdot \sin \theta-r) \cdot d x \cdot d y
$$

Using the inverse Fourier transform, the inverse Radon transform formula can easily be derived:

$$
\mu(x, y)=\frac{1}{2 \cdot \pi} \int_{0}^{\pi} g_{\theta}(x \cdot \cos \theta+y \cdot \sin \theta) \cdot d \theta
$$

Where $g_{\theta}$ is the derivative of the Hilbert transform $p_{\theta}(r)$.

Atmospheric turbulence is a highly important issue in solar observation. In the lowest areas of the atmosphere, atmospheric turbulence varies greatly from night to day, and is heavier during daylight hours. One of its main causes is solar radiation. Air is heated by solar radiation, and subsequently the hottest and lightest layers of air rise while the coolest layers replace them before falling due to their greater density. The number of turbulent layers in the atmosphere is variable. Let $N_{l}$ be the total number of turbulent layers, each one with a different height value. The aperture-plane phase of light coming from the Sun in the direction $\theta=\left(\theta_{r}, \theta_{y}\right)$ for a certain time $t$ can be expressed as follows [4]:

$$
y(\theta, t)=\sum_{i=1}^{N_{l}} P_{\theta}^{i} \emptyset_{i}(t)
$$

Where:

$\emptyset_{i}(t)$ represents a column vector of a phase distortion on a discrete grid for the $\mathrm{i}^{\text {th }}$ turbulence layer.

$P_{\theta}^{i}$ is a ray-tracing submatrix which extracts a phase distortion.

A tomographic reconstruction is determined to minimize the aperture plane phase variance for each direction $\theta_{k}[5]$ : 


$$
E_{\theta k}=\operatorname{argmin}\left\langle\left\|y_{k}-y_{k}\right\|^{2}\right\rangle
$$

Where $E_{\theta k}$ is the tomographic reconstruction for the direction $\theta_{k}, y_{k}$ is the actual aperture-plane phase coming from the direction $\theta_{k}$ and $<>$ indicates the ensemble average over time.

\subsection{Adaptative optics.}

When the light wavefront that comes from the Sun penetrates the atmosphere, it suffers some distortions caused by a series of optically-turbulent layers present at different altitudes and with different relative strengths. This turbulence changes the shape and morphology of the wavefront . Therefore, in order to obtain a goodquality image it is necessary to correct the error induced. The incoming wavefront is corrected by applying adaptive optics techniques [6]. In the case of the present research, it is done with the help of a Multi-Conjugate Adaptive Optics System (MCAO) [7].

Adaptive optics $(\mathrm{AO})$ is a technology used to improve the performance of optical systems by reducing the effect of incoming wavefront distortions. This is achieved by deforming a mirror in order to compensate for the distortion [8]. The main purpose of adaptive optics, which was first used in the 1980s, is to correct the aberrations caused by the atmosphere in the wavefront.

Fig. 2 shows the main elements of a closed-loop adaptive optics imaging system. As can be observed in the flowchart, light coming from the sun reaches the telescope. A certain amount of the light received is sent to the wave-front sensor (WFS). Thanks to a deformable mirror that is employed as a corrector, and with the help of a computer, the image is adjusted.

To characterize the incoming wave-front received by large telescopes, it is common to use the Shack-Hartman Wavefront Sensor (SH-WFS) [9]. This sensor is composed of several lenses with the same focal length which are focused on different photon sensors. The resulting wave-front can be split into a matrix of tilts, and the deviation from the focal spot can be calculated. The SH-WFS is an evolution of the Hartmann mask [10] and its main goal is to improve the images of ground telescopes.

When speaking about solar adaptive optics, there are certain differences from night observation that must be considered. Firstly, and due to its distance to planet earth, the Sun can be considered as a massive, extremely fast-moving object. Furthermore, the solar telescope suffers the effects of radiation from the Sun and the image quality is affected by atmospheric turbulence.

The photons of the observed image, in this case a portion of the solar surface, pass through the WFS matrix lens showing as many different images as there are subapertures, all very similar but different due to the influence of atmospheric 
turbulence. With the help of the well-known Fractal Iterative Method (FrIM) algorithm [11], the best image is selected and linked to the rest of images to assess how the turbulence affects them. The output of this stage is a slopes matrix and a reconstruction algorithm is subsequently applied.

In the case of the present research, Multiconjugate Adaptive Optics (MCAO) are applied. MCAO utilizes more than one (and more usually three or four) deformable mirrors (DM) in the process of correcting the wave front aberrations. Each mirror is optically conjugated to an individual distance from the telescope and corrects the aberrations produced by different layers of atmosphere.

The reconstruction technique proposed in this research is a MCAO based on a Convolutional Neural Network (CNN). The foundations of CNNs are explained in the next section.

The reconstruction performance is measured by means of the Root Means Square Wave-front Error (RMSE WFE). It is calculated as follows:

$$
R M S E W F E=\sqrt{\bar{W}^{2}-[\bar{W}]^{2}}
$$

Where the wave-front is represented by $W(x, y), \bar{W}^{2}$ is the average of the squared wave-front deviations that can be expressed as $\bar{W}^{2}=\frac{1}{A} \int X(x, y)^{2} d x d y$ and where $A$ is the area of the pupil and $[\bar{W}]^{2}$ is the square of average wavefront deviation [12].

\subsection{Convolutional neural networks.}

Convolutional neural networks were developed in order to deal with wellstructured input with strong spatial dependencies. One of the most common examples of this kind of structures are pictures. The first use of a convolutional neural network was reported by LeCunn et al. in 1998 [13]. The main idea behind convolutional neural networks involves flattening images in order to make it possible to store them as vectors. Fig. 3 shows an example in which a 4 by 4 image is flattened to a vector. Afterwards, it is assigned to a neuron. In general, a convolutional neural network can be defined as a neural network that has one or more convolutional layers.

A convolutional layer takes a 2D array that generally represents an image and passes a logistic regression over all the elements of the array. Please note that in general these input arrays have lengths such as $4(2 \times 2), 9(3 \times 3)$, or $16(4 \times$ 4). In most cases, as the information comes from an image, data values are in the range from 0 to 255 . The output vector generated will typically have a lower dimension than the original one.

It is not always necessary to move the local receptor field one component at a time. In fact, the number of parameters that we move the receptive field between taking inputs is called the stride of the convolutional layer. This parameter is important, as it determines the dimensional reduction that will be performed by 
the convolutional layer. Fig. 4 shows a simple example with a $4 \times 42 \mathrm{D}$ convolutional layer, in this case, using a $2 \times 2$ receptive field. Therefore, the output will be $3 \times 3$.

Each layer of a convolutional neural network has the following three dimensions: height, width and depth. Please note that in this context, depth refers to the number of channels of the layer. The convolution operation from $\mathrm{q}^{\text {th }}$ layer to $(q+1)^{\text {th }}$ can be formally defined as follows:

$$
h_{i j p}^{q+1}=\sum_{r=1}^{F_{q}} \sum_{s=1}^{F_{q}} \sum_{k=1}^{d_{q}} w_{r s k}^{(p, q)} h_{i+r-1, j+s-1, k}^{(q)}
$$

Taking into account that such an expression is valid for: $\forall i \in\left\{1 \ldots, L_{q}-F_{q}+1\right\}$, $\forall j \in\left\{1, \ldots, B_{q}-F_{q}+1\right\}$ and $\forall k \in\left\{1, \ldots d_{q}+1\right\}$

This convolutional operation is applied in the formula above to the $\mathrm{p}^{\text {th }}$ filter in the $\mathrm{q}^{\text {th }}$ layer. Their parameters are denoted by the 3-dimensional tensor:

$$
W^{(p, q)}=\left[w_{i j k}^{(p, q)}\right]
$$

Where $i$ is the position along height, $j$ the position along width and $k$ the position along depth in the filter considered. The feature maps in the $\mathrm{q}^{\text {th }}$ layer are represented by the following tensor: $H^{(q)}=\left[h_{i j k}^{(q)}\right]$ please note that it also has three dimensions. The formula is a dot product over the entire volume of the filter, which is repeated for all the spatial positions.

The training of a convolutional neural network may be performed rapidly as there are only a few parameters to train. This training consists of training the local receptive fields of the layers, their bias and weights. The most commonlyemployed activator function is called a rectified linear unit (ReLu) $[14,15]$. It can be defined as follows:

$$
\rho(x)=\max (x, 0)
$$

which means that this activation function will return the value of $x$, unless it is negative, in which case it will return a 0 . In many applications, colour images are processed. This means that three different matrixes are available for the image, one for each of the RGB channels. Please note that this is not the case in the present research, where grey scale images are employed and only one matrix is required.

In the case of the present research, not only ReLu activation functions are employed, but also Leaky Rectified Linear Units (Leaky ReLu). They are expressed by the following function:

$$
L \rho(x)=\left\{\begin{array}{c}
x \text { if } x>0 \\
0.01 \cdot x \text { otherwise }
\end{array}\right.
$$


In general, it can be said that the computation of Leaky ReLu is the faster of the two. Furthermore, and according to previous research [16], in order to discover which is the most convenient, it must be tested at each application. An advantage of ReLu over Leaky ReLu is that it is not necessary to think about the value of the negative slope.

In order to add generalization after a ReLu or Leaky ReLu layer, it is possible to employ the local response normalization. This methodology creates a competition among different filters. Let be $\mathcal{L}$ a layer formed by a total of $n$ filters whose activation values for coordinates $(x, y)$ are $\left\{a_{1}, \ldots a_{n}\right\}$. It is possible to transform those values into normalized ones that will be noted as $a_{i}$ by means of the following equation:

$$
b_{i}=\frac{a_{i}}{\left(k+\alpha \sum_{j=1}^{n} a_{i}^{2}\right)^{\beta}}
$$

In the case of the present research, the following previously-empirically-adjusted values [17] are employed: 2 for $k, 10^{-4}$ for $\alpha$ and 0.75 for $\beta$. Nevertheless, it also would be possible to normalize the values with the help of a more general method such as Box-Cox or Johnson transformations.

The max pooling process is a sampled-based discretization process. Its aim is to perform the image dimensional reduction. When using max-pooling, it is assumed that important information is contained in adjacent pixels. This principle, although not very useful for dealing with real-world images, is interesting for feature maps.

In a convolutional neural network, max-pooling and convolutional layers are combined. After passing the original information through some layers, the processed information can be used as input data for a classification model such as logistic regression.

The reconstruction algorithm presented in this research, based on CNN, is trained using images of the Sun in different turbulence conditions. The WFS images are the input data and the output is the voltage of the actuators that modify the shape of the deformable mirror surface to get a perfect reconstruction. 8,000 images of the Sun were used for the training, together with thousands of different turbulence profiles.

The real-time tomographic reconstructor developed is able to correct the error introduced by the atmosphere in the light wave-front received from the Sun. The $\mathrm{CNN}$ was trained and validated using data from the Durham AO Simulation Platform (DASP) as input information. This Platform has been specifically adapted for this project in order to simulate a solar telescope.

\section{Results and discussion.}

The input information employed for training the model consists of a set of 1,000 different images of the sun created with the help of the Durham Adaptive Optics Simulation Platform (DASP) [18]. Each of these images represents a different 
area of the Sun's surface. Please note that although all the images are different, overlap is possible. In other words, it is possible for a certain area of the solar surface to be present in more than one image. Fig. 5 shows one of the images of the Sun used for the DASP simulator. A Monte-Carlo modelling tool is employed for the simulation of solar $\mathrm{AO}$ systems. The information employed in the training of the CNN contains images that correspond to different turbulence profiles. In order to configure these profiles, the following parameters are modified: turbulence energy $\left(R_{0}\right)$, wind speed $\left(v_{0}\right)$ and turbulence height $\left(H_{0}\right)$ from 0 to 15 kilometres in steps of 50 meters. This means that each of the images goes through a total of 300 different height turbulence profiles. Please note that each turbulence profile is simulated 10 times. Therefore, the training set has a total of three million different images.

In a traditional AO system, the first step involves obtaining the centroids of the images of all the sub apertures. The image that is closest to the original is selected, and the rest of the images are correlated. Afterwards, these centroids are employed for the tomographic reconstruction.

In the present research, which makes use of deep learning methodologies, the process follows a flowchart like what is detailed in Fig. 6. The information from the Shack-Hartmann wavefront sensors (Fig. 7) is employed as input images for the reconstructor based on convolutional neural networks.

One of the main challenges of $\mathrm{AO}$ in solar observation is that the Sun is a massive object for a telescope. This means that only a portion of the Sun can be seen with a telescope at any given moment and this portion covers all the subaperture of the sensor. This is a great difference when compared with night observation, where a star is seen as a light spot which after aberrated by the turbulence profile becomes a cloud of points in the subaperture of the sensor.

All the Shack-Hartmann wavefront sensors receive the same image but with certain differences due to turbulence in the atmosphere. In the case of the model training not only do we have the image deformed by turbulence, but also the original image of the Sun without turbulence. Therefore, we will be able to train a system capable of predicting how the deformable mirror must behave in order to compensate for atmospheric turbulence and obtain a clear image of the Sun.

In traditional multi-layer perceptron ANN-AO systems each subaperture is processed separately [10] However, in our case, as we use CNN, the images of all the subapertures are processed together and the features extracted successively in each convolutional filter.

As was stated before, one of the strengths of CNNs is that they are very good at dealing with information whose spatial location is relevant. In the case of the present research, the output values of the CNN are the voltage figures of the actuators in the deformable mirror. In other words, the target of the CNN is to obtain the voltage values that modify the deformable mirror in such a way that 
the image that it reflects compensates for atmospheric turbulence and happens to be the inverse of the turbulence profile which aberrated the solar image.

Simulations were performed applying different offsets to the images. The computer-simulated Shack-Hartmann wavefront sensors have $10 \times 10$ sub apertures (please see Fig. 7) with $28 \times 28$ pixels each. The deformable mirror simulated has a total of 117 actuators.

The CNN made use of different numbers of convolutional layers, from four to nine, and kernels of $3 \times 3,5 \times 5$ and $7 \times 7$. The activation function were ReLu and Leaky ReLu. Afterwards, $5 \times 5$ maxpooling was applied in all cases.

The input layer of the neural network was formed by the pixel output of the last layer of the CNN. This neural network was a multilayer perception, with only one hidden layer, which in all the cases trained for the present research had 1024 neurons.

\begin{tabular}{llcccc}
\hline \multicolumn{1}{c}{ Layer } & \multicolumn{1}{c}{ Parameter } & Values & Units \\
\hline Common & Test name & test1 & test2 & test3 & \\
\hline \multirow{2}{*}{ Ground } & Altitude & 0 & 0 & 0 & $\mathrm{~m}$ \\
layer & Wind Speed & 7.5 & 7.5 & 10 & $\mathrm{~m} / \mathrm{s}$ \\
& Wind direction & 0 & 0 & 0 & degrees \\
\hline
\end{tabular}

First layer

\begin{tabular}{lcccc} 
Altitude & 4,000 & 2,500 & 6,500 & $\mathrm{~m}$ \\
Wind Speed & 12.5 & 12.5 & 15 & $\mathrm{~m} / \mathrm{s}$ \\
Wind direction & 330 & 330 & 330 & degrees \\
\hline
\end{tabular}

Second layer

$\begin{array}{lcccc}\text { Altitude } & 10,000 & 4,000 & 10,000 & \mathrm{~m} \\ \text { Wind Speed } & 15 & 15 & 17,5 & \mathrm{~m} / \mathrm{s} \\ \text { Wind direction } & 135 & 135 & 135 & \text { degrees }\end{array}$

Third layer

$\begin{array}{lcccc}\text { Altitude } & 15,500 & 13,500 & 15,500 & \mathrm{~m} \\ \text { Wind Speed } & 20 & 20 & 25 & \mathrm{~m} / \mathrm{s} \\ \text { Wind direction } & 240 & 240 & 240 & \text { degrees }\end{array}$

Table 1. Parameters of the three turbulent cases considered to be like those that can be found at IAC.

As was stated before, the training was performed using only one turbulent layer for each image in each case. For the validation of the trained models, Montecarlo Simulation was employed in order to generate three different atmospheric profiles that would be like the real ones to be found at the facilities of the Instituto Astrofísico de Canarias (IAC), on the island of La Palma (Canary Islands, Spain). 
These three profiles, considered as good, medium and bad, have already been employed in previous research. These three cases made use of four different turbulent layers, with each one being of different height, wind speeds and directions. Their parameters are detailed in Table 1.

Table 2 and 3 show the normalized errors of the deformable mirror commands for ReLu and Leaky ReLu kernel functions respectively for the three test scenarios. As may be observed, in general, it can be said that the greater the number of layers, the smaller the error. Also, smaller errors are obtained with $3 \times$ 3 kernel when compared with $5 \times 5$ and $7 \times 7$. Please note that the minimum error value is 0.18842 and is obtained when 8 layers are employed with $3 \times 3$ kernels.

\begin{tabular}{|c|c|c|c|c|c|c|c|}
\hline & & 4 layers & 5 layers & 6 layers & 7 layers & 8 layers & 9 layers \\
\hline & $3 \times 3$ kernel & 0.211543 & 0.201279 & 0.195589 & 0.187791 & 0.188420 & 0.190041 \\
\hline \multirow[t]{2}{*}{ Test 1} & $5 \times 5$ kernel & 0.213286 & 0.203987 & 0.202882 & 0.193617 & 0.192800 & 0.194285 \\
\hline & $7 \times 7$ kernel & 0.218320 & 0.208755 & 0.203547 & 0.197540 & 0.194529 & 0.197858 \\
\hline \multirow{3}{*}{ Test 2} & $3 \times 3$ kernel & 0.219107 & 0.219644 & 0.201318 & 0.188881 & 0.191916 & 0.204941 \\
\hline & $5 \times 5$ kernel & 0.228367 & 0.206752 & 0.213619 & 0.195264 & 0.204193 & 0.213279 \\
\hline & $7 \times 7$ kernel & 0.235252 & 0.215690 & 0.221186 & 0.209351 & 0.202488 & 0.213410 \\
\hline \multirow{3}{*}{ Test 3} & $3 \times 3$ kernel & 0.220384 & 0.230254 & 0.212205 & 0.200777 & 0.193051 & 0.215512 \\
\hline & $5 \times 5$ kernel & 0.235567 & 0.213712 & 0.218565 & 0.209500 & 0.216587 & 0.218077 \\
\hline & $7 \times 7$ kernel & 0.244911 & 0.228288 & 0.230779 & 0.212701 & 0.215510 & 0.229981 \\
\hline
\end{tabular}

Table 2. RMSE WFE of the deformable mirror commands (kernel function: ReLu).

\begin{tabular}{|c|c|c|c|c|c|c|c|}
\hline & & 4 layers & 5 layers & 6 layers & 7 layers & 8 layers & 9 layers \\
\hline & $3 \times 3$ kernel & 0.138543 & 0.104979 & 0.168089 & 0.232991 & 0.18132 & 0.106141 \\
\hline \multirow[t]{3}{*}{ Test 1} & $5 \times 5$ kernel & 0.252486 & 0.216387 & 0.285682 & 0.148517 & 0.277 & 0.231985 \\
\hline & $7 \times 7$ kernel & 0.14472 & 0.291255 & 0.227547 & 0.29614 & 0.114829 & 0.247358 \\
\hline & $3 \times 3$ kernel & 0.152110 & 0.111050 & 0.170712 & 0.233598 & 0.183859 & 0.111347 \\
\hline \multirow[t]{3}{*}{ Test 2} & $5 \times 5$ kernel & 0.265687 & 0.234299 & 0.301850 & 0.157323 & 0.303653 & 0.242232 \\
\hline & $7 \times 7$ kernel & 0.154358 & 0.294695 & 0.246192 & 0.298045 & 0.121044 & 0.255289 \\
\hline & $3 \times 3$ kernel & 0.158442 & 0.111950 & 0.173266 & 0.254239 & 0.198599 & 0.113427 \\
\hline \multirow[t]{2}{*}{ Test 3} & $5 \times 5$ kernel & 0.277282 & 0.255696 & 0.321212 & 0.157395 & 0.320119 & 0.261627 \\
\hline & $7 \times 7$ kernel & 0.158055 & 0.303917 & 0.261078 & 0.301766 & 0.131963 & 0.260473 \\
\hline
\end{tabular}

Table 3. RMSE WFE of the deformable mirror commands (kernel function: Leaky ReLu).

According to the results of the Anderson-Darling normality test, the values of the RMSE WFE do not follow a normal distribution $(A D=1.163, p<0.005)$. This lack of normality in the data led us to the use of a no-parametric test for the data analysis. The application of the Kruskal-Wallis test in order to find differences in the RMSE WFE values according to the kernel function allows us to say that there are no statistically-significant differences in the median among groups when kernel groups are compared $(H=0.73, d f=1, p=0.393)$. The results are also equivalent for the different profiles employed for the validation $(H=5.17, d f=2$, 
$p=0.075)$, although in this case the $\mathrm{p}$-value is closer to the significant cutting point. The kernel dimension gives statistically-significant differences $(H=20.93$, $d f=2, p<0.001)$, giving a lower median value for the $3 \times 3$ kernel $(0.190979)$, followed by the $5 \times 5$ kernel $(0.217332)$ and the the $7 \times 7$ kernel $(0.219753)$. The number of layers employed does not show statistically-significant differences ( $H=3.98, d f=5, p=0.553$ ) according to the results of the test. Fig. 8 shows a boxplot of the RMSE WFE values by kernel dimension, and allows us to see how RMSE WFE values are lower in $3 \times 3$ kernels.

\section{Conclusions.}

In the present research, a learning algorithm able to train the CNN was developed. As far as the researchers know, this is the first time that an $\mathrm{AO}$ system based on CNN has been developed for solar telescopes. The CNN reconstructor obtained from the training has been validated in the simulated environment. The results obtained with the two kernels tested are very similar, and the only differences found were due to the kernel dimension. Although this project is at a very early stage, from our point of view, the results are promising, and we can say that the combination of deep learning and $A O$ is able to provide highperformance results.

The next step will be to test the reconstructor in a real system. Please note that the final goal of this research is to have a system ready to be employed in the European Solar Telescope, a future 4-meter class aperture device.

Furthermore, the developments made for the purposes of solar observation would also be of interest to the field of ophthalmology.

\section{Acknowledgements.}

This research was funded by MINISTERIO DE ECONOMIA INDUSTRIA $Y$ COMPETITIVIDAD, grant number MINECO-18-AYA2017-89121-P. We would like to thank Anthony Ashworth for his revision of the English grammar and spelling of the manuscript.

\section{References.}

1. Vaquero J, Gallego M. (2014) Reconstructing past solar activity using meridian solar observations: The case of the Royal Observatory of the Spanish Navy (1833-1840). Adv in Space Res, 53 (8), 1162-1168.

2. Moore P. The data bank of astronomy. Bristol: Institute of Physics Publishing; 2011.

3. Helin, T., Kindermann, S., and Saxenhuber, D. (2016) Towards analytical model optimization in atmospheric tomography. Mathematical Methods in the Applied Sciences, 40 (4), 1153-1169. 
4. Ono YH, Akiyama M, Oya S, Lardiére O, Andersen DR, Correia C, Jackson K, Bradley C (2016). Multi time-step wavefront reconstruction for tomographic adaptive-optics systems. J Opt Soc Am A Opt Image Sci Vis. 33(4):726-40.

5. Correia, C., Jackson, K., Véran, J.-P., et al. (2013) Static and predictive tomographic reconstruction for wide-field multi-object adaptive optics systems. Journal of the Optical Society of America A, 31 (1), 101.

6. AG Basden, D Atkinson, NA Bharmal, U Bitenc, M Brangier, T Buey, T Butterley, D Cano, F Chemla, P Clark, M Cohen, J-M Conan, FJ De Cos, C Dickson, NA Dipper, CN Dunlop, P Feautrier, T Fusco, JL Gach, E Gendron, D Geng, SJ Goodsell, D Gratadour, AH Greenaway, A Guesalaga, CD Guzman, D Henry, D Holck, Z Hubert, JM Huet, A Kellerer, C Kulcsar, P Laporte, B Le Roux, N Looker, AJ Longmore, M Marteaud, O Martin, S Meimon, C Morel, TJ Morris, RM Myers, J Osborn, D Perret, C Petit, H Raynaud, AP Reeves, G Rousset, F Sanchez Lasheras, M Sanchez Rodriguez, JD Santos, A Sevin, G Sivo, E Stadler, B Stobie, G Talbot, S Todd, F Vidal, EJ Younger (2016) Experience with wavefront sensor and deformable mirror interfaces for wide-field adaptive optics systems. Mon. Not. R. Astron. Soc. 459 (2): 1350-1359.

7. Soltau D, Berkefeld T, Sanchez Capuchino J, Collados Vera M. del Moro D, Löfdahl M., Scharmer G. Adaptive Optics and MCAO for the 4-m European Solar Telescope EST, Proceedings SPIE 2010, 7736. doi: $10.1117 / 12.856851$.

8. Tyson, R.K. (1991) Adaptive Optics Systems. Principles of Adaptive Optics, 53-100.

9. Schillinger, M., Delbru, F., Meisse, C., and Hours, D. (2008) Aladin Telescope stability verification using a Hartmann wavefront sensor. Proc. SPIE 7003, Optical Sensors 2008, 70031H.

10. de Cos Juez, F.J., Sanchez Lasheras, F., Roqueñí, N., Osborn, J. (2012) An ANN-Based Smart Tomographic Reconstructor in a Dynamic Environment. Sensors, 12 (7), 8895-8911.

11. Béchet, C., Tallon, M., and Thiébaut, E. (2006) FRIM: minimum-variance reconstructor with a fractal iterative method. Advances in Adaptive Optics II.

12. Deng, L. (1995) Theory and experiment on a correlation wavefront sensor for extended target adaptive optics corrections. Optical Engineering, 34 (12), 3488.

13. Lecun, Y., Bottou, L., Bengio, Y., and Haffner, P. (1998) Gradient-based learning applied to document recognition. Proc. of the IEEE, 86 (11), 2278-2324.

14. Skansi S. Introduction to Deep Learning. From logical calculus to Artificial Intelligence. Basel (Switzerland): Springer International Publishing AG; 2018.

15. Galan CO, Sanchez Lasheras F, de Cos Juez FJ, Bernardo Sánchez AB. (2017) Missing data imputation of questionnaires by means of genetic algorithms with different fitness functions. J Comput Appl Math, 311, 704717. 
16. Kuzovkin, I, Vicente, R, Petton, M, Lachaux JP, Baciu M, Kahane P, Rheims S, Vidal JR, Aru J (2018) Activations of Deep Convolutional Neural Network are Aligned with Gamma Band Activity of Human Visual Cortex. Communications Biology; 1 (107)

17. Aggarwal CC. Neural Networks and Deep Learning. Berlin: Springer; 2018.

18. Basden, A., Bharmal, N., Jenkins, D., et al. (2018) The Durham Adaptive Optics Simulation Platform (DASP): Current status. SoftwareX, 7, 63-69. 
Figures.

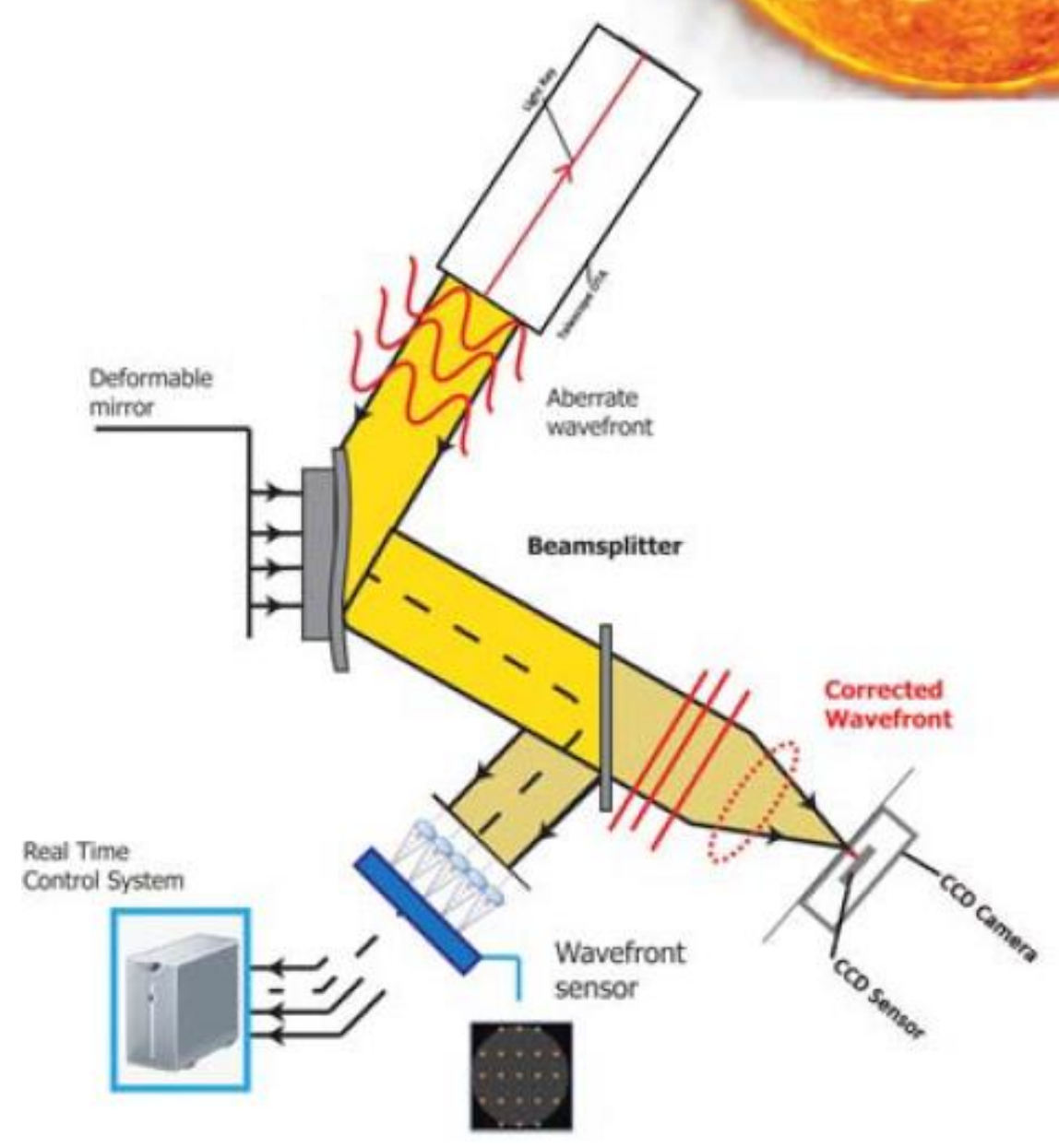

Figure 1. Conventional configuration of a closed-loop AO imaging system. 


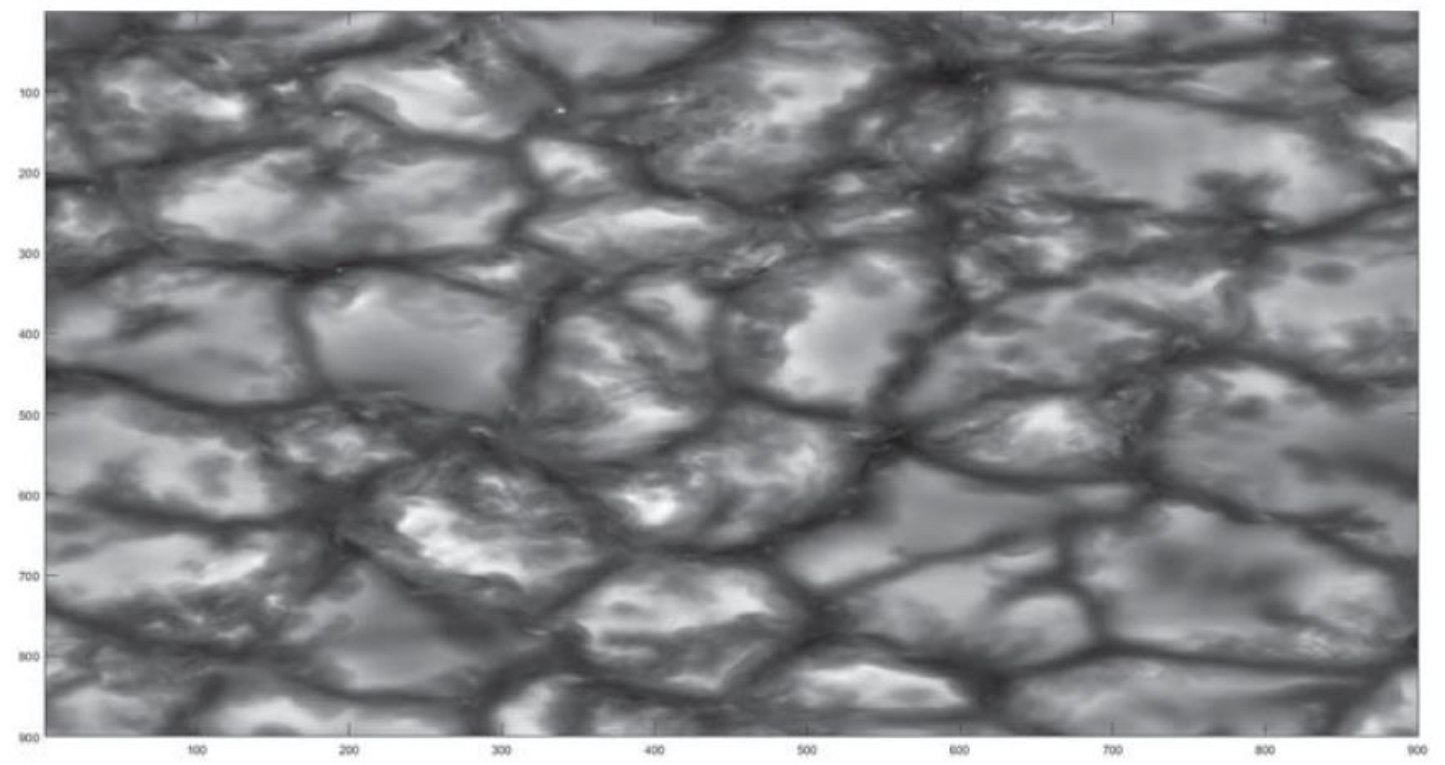

Figure 2. Solar image used for simulation in DASP.

4 to 7 convolutional layers

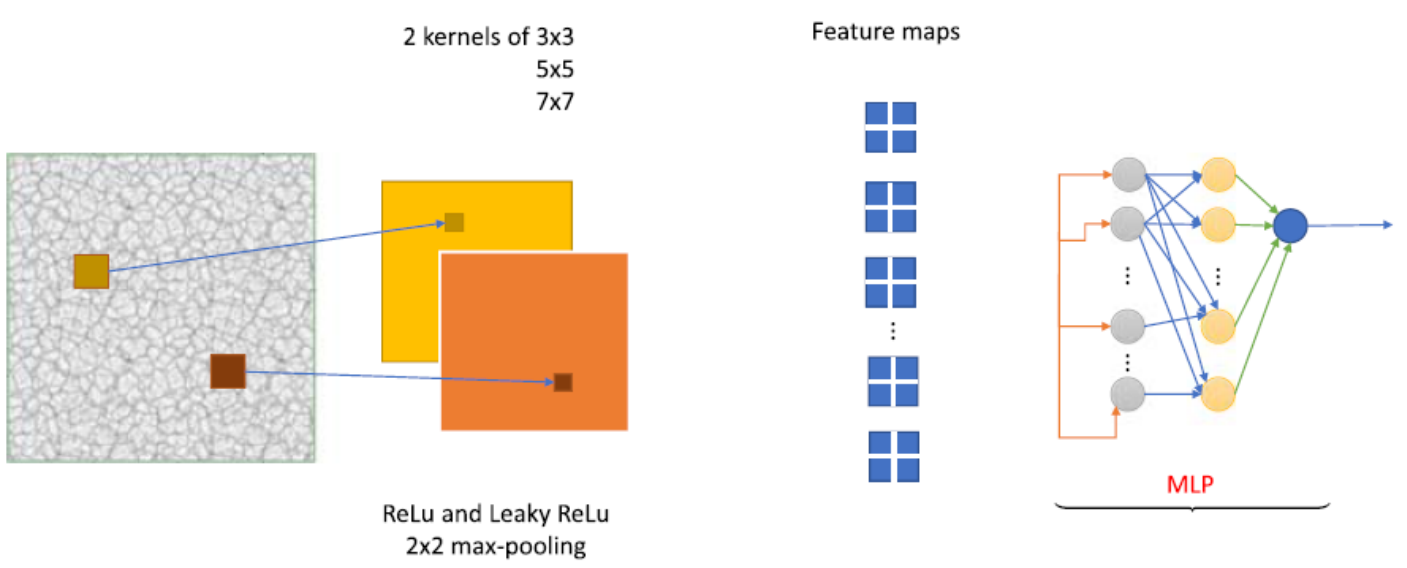

Figure 3. Flowchart of the process. 


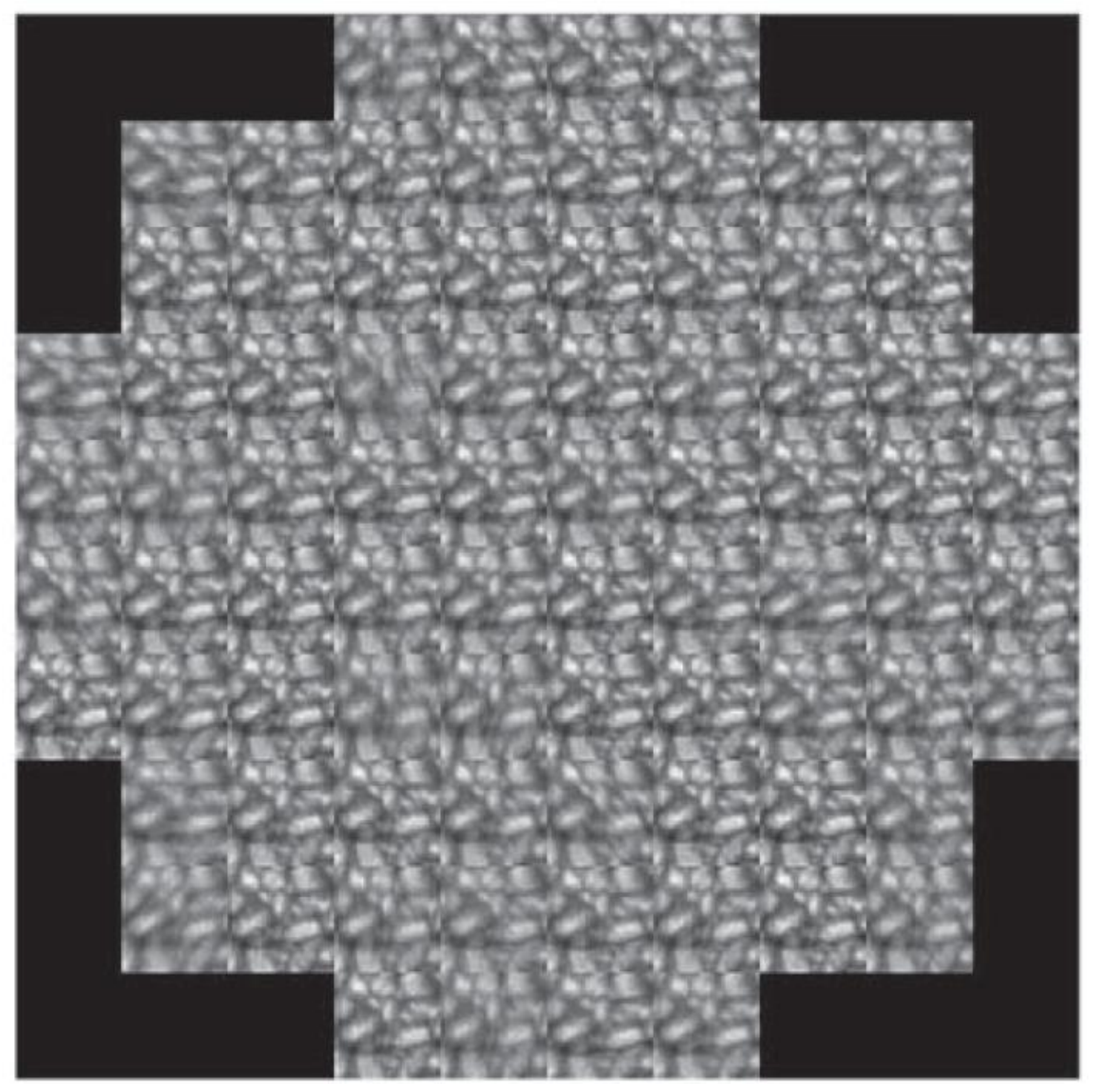

Figure 4. Image obtained with the information of the Shack-Hartmann wavefront sensors. 


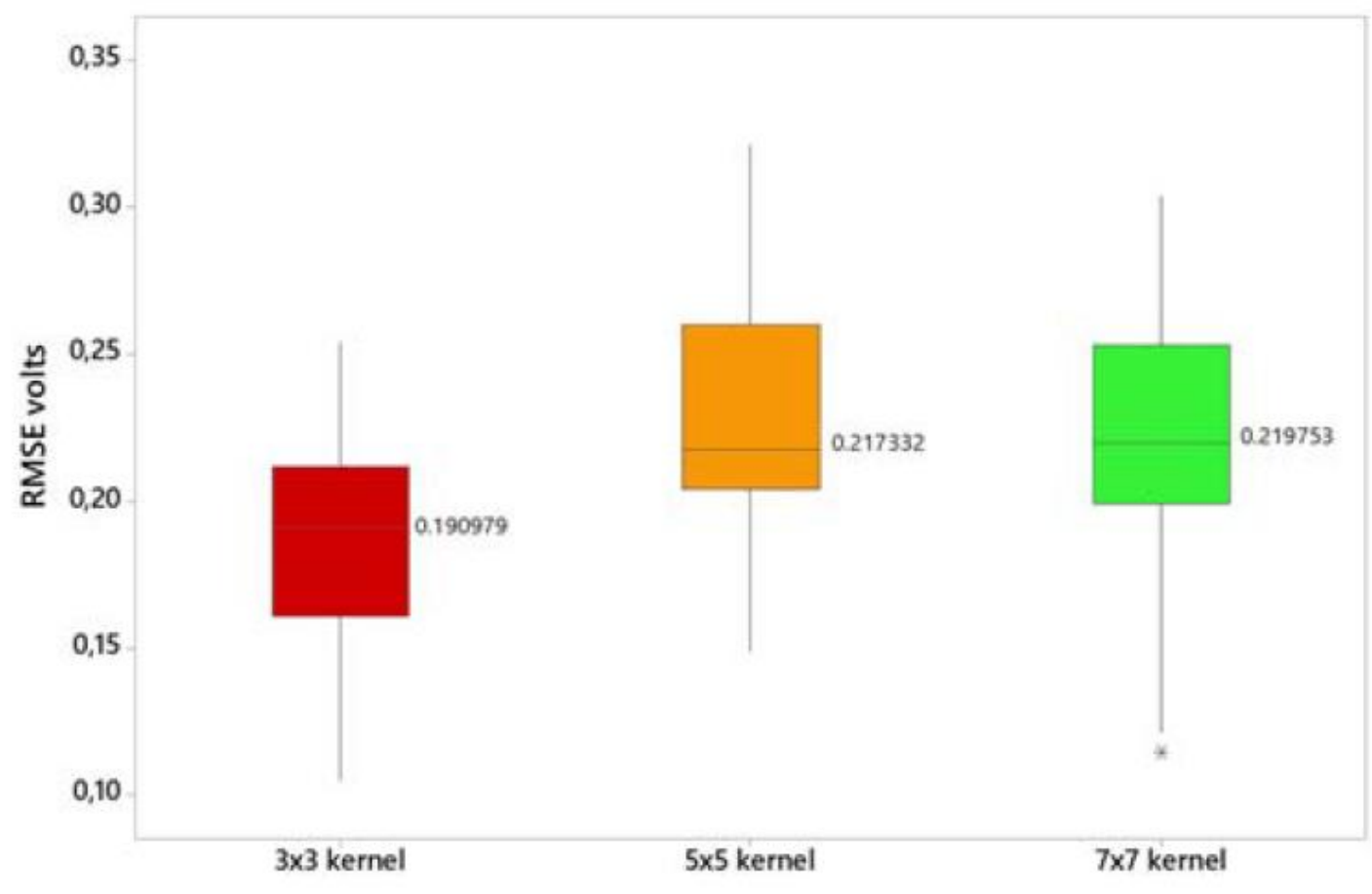

Figure 5. Boxplot of the RMSE WFE values by kernel dimension. 